\title{
Normative values for physical fitness in children aged 11-17 in Kosovo
}

\author{
Milaim Berisha ${ }^{1 \mathrm{ABCDE}}$, Murat Çillii ${ }^{2 \mathrm{ACDE}}$ \\ ${ }^{1}$ Institute of Educational Sciences, Faculty of Sports Science, Sakarya University, Turkey \\ ${ }^{2}$ Department of Coaching Education, Faculty of Sports Science, Sakarya University, Turkey
}

Authors' Contribution: A - Study design; B - Data collection; C - Statistical analysis; D - Manuscript Preparation; E - Funds Collection.

\begin{abstract}
Purpose: In the present study, the purpose was to determine the normative values for physical and biomotoric characteristics of children aged 11-17 in Kosovo.

Material: $\quad 742$ students who were selected according to the Random Sampling Method from 24 schools determined in the cities and rural areas in all regions were included in the study ( $n=347$ girls, $n=395$ - boys). Volunteering students who did not have any acute or chronic disease that might avoid testing were included in the study after the permissions were received from the Ministry of National Education, Principals of the Schools, and the support of the parents. The declarations of Helsinki were taken into consideration in the study.

Results: The results of each test were given as the norm values according to gender and age together with $20 \%$ groups, which is considered as the Canadian Standard. The data on the physical and biomotoric characteristics of the children living in Kosovo were compared with the data of the World Health Organization and the literature data of other countries.

Conclusions: While the children living in Kosovo showed higher performances in the tests that were affected by anthropometric properties like height and body fat percentage and by genetic properties like the speed of the movements of the arms when compared with the children from other countries from the same age group; they had lower performances in the tests that were affected by life style and physical activity levels like endurance, long jump, and flexibility.

Keywords: Norm Values, Physical Fitness, EuroFit, Kosovo.
\end{abstract}

\section{Introduction}

Making comparisons is necessary in making the values like adequate, low, very good, perfect or normal be acquired for each feature that is measured, and certain criteria are needed in making comparisons. The models, criteria or standards that are accepted officially are called norms $[1,2]$. A boy whose height is measured as $1.70 \mathrm{~m}$ may be considered to be short in Sweden; however, the same boy may be considered to be very tall in Kenya. A girl who has the best Standing Long Jump value at a school as the best degree of the school may be assessed as being below the normal values when her explosive power properties are compared with another girl from another school. On the other hand, if a child has Maximum Oxygen Use Capacity below the normal values, s/he may be considered to have a chronic disease. It is known that as well as gender and genetic factors, the factors like nutrition, metabolic diseases, chronic diseases or physical activity levels have certain effects on the physical, spiritual and cognitive developments of children and young people. In addition to this, the adequacy in short and long distance running, push-up, sit-up, jumping and similar motoric characteristics show the fitness in good health [3]. The probability is higher for children with low functional, anthropometric and motor properties to face health problems in their future lives (high blood pressure, diabetes mellitus, cancer, excessive fatness and cardiovascular diseases, etc.) $[3,4]$. Especially by determining

(c) Milaim Berisha, Murat Çilli, 2018

doi:10.15561/18189172.2018.0103 the norms of the physical and motoric properties of children at development period, it becomes possible to diagnose and eliminate possible problems at earlier ages $[5,6]$. On the other hand, it is also important to define the norm values in the selection of the aptitudes [7]; planning of physical education and sports classes [8-10]; and in performing international comparative studies [11].

In 1956, in European countries, the Physical and Sports Council was formed, and motor and anthropometric testes were used to follow-up the health status of school age children between the ages 6-18 [12-14]. The tests that are applied under the umbrella terms of Eurofit Test Battery have been used in many European countries especially on children, and certain criteria have been defined for different countries. Physical and motoric properties, which may be affected by the geographical area, culture, genetic and many similar factors, may vary not only among countries but also according to the properties of the area where people live $[15,16]$. Although the literature that define the national, regional and international growth percentiles is improving with each passing day, there are no studies conducted in Kosovo, which has one of the youngest population in European Zone [17, 18], on the development and physical fitness status of the children. In the present study, the purpose was to determine the norm values of the physical and biomotoric properties of the students between 11-17 years of age in Kosovo according to age and gender, and to compare these data with the World Health Organization data and the literature of other countries. 


\section{Material and methods}

Participators. Nearly 300 thousand students between the ages 11-17 are receiving education in Kosovo, which consists of 30 cities located in 7 geographical regions [18]. 742 students who were selected according to the Random Sampling Method from 24 schools determined in the cities and rural areas in all regions were included in the study. 347 of the participants were female and 395 were male (Figure 1). Volunteering students who did not have any acute or chronic disease that might avoid testing were included in the study after the permissions were received from the Ministry of National Education, Principals of the Schools, and the support of the parents. The declarations of Helsinki were taken into consideration in the study.

The Distribution of the Sampling of the Study according to Cities and Regions (Figure 1).

Eurofit Physical Fitness Test Battery is a test set consisting of 9 physical fitness tests that involve flexibility, speed, endurance and power. The Standard Test Battery, which was developed by the European Council for school age children, has been used in many schools in Europe since 1988 [19]. The battery was designed to be completed within 35-40 minutes with very simple equipment, and is fairly adequate to determine the physical and motoric status of children $[12,14]$. The Height (L), Weight (W), Body Mass Index (BMI), Flamingo Balance (FLB), Plate Tapping (PLT), Sit and Reach (SAR), Standing Broad Jump (SBJ), Hand Grip (HGR), Sit Up (SUP), Bent Arm Hang (BAH), Shuttle Run 10x5 meters (SHR), $20 \mathrm{~m}$ Shuttle Run (ESHR) tests, which were included in the
Eurofit Test Battery, were also included in the present study [19]. In addition to the Eurofit Physical Fitness Test Battery, the Body Fat Percentage (BF\%), Arch Up Test (AUT), Standing Medicine Ball Throw (MBTH), Push-up (PU), Squat Jump (SJ), Countermovement Jump (CMJ), Maximal Anaerobic Power (MAP), 30-Meter Speed Test $(30 \mathrm{mST})$, Maximum Oxygen Intake $\left(\mathrm{VO}_{2}\right.$ max $)$ tests were also employed in the study. All these tests, which were included in the study, were chosen by considering the factors like muscle groups, joint angles, contraction types, contraction speeds, movement models according to the body parts [20].

The Materials used in Measuring the Physical and Motoric Properties

In measuring the heights of the students, a Stadiometer with $1 \mathrm{~cm}$ sensitivity was used. In determining the Body Weight, Body Mass Index and Body Fat Percentage values, the TANITA Segmental Body Composition BC-545N was used. The hand grip power was measured with a standard hydraulic hand dynamometer (Baseline 12-0240). Situp test, Reverse Shuttle on the Case Test, Push-up tests were assessed with repetition numbers. In FBT, PLT, SUP and SHR tests, the "QQ Japan, HS45 10 Lap Memory" chronometer was used. A mat fixed to the ground with marked distances and a medicine ball weighing $4 \mathrm{~kg}$ were used in Standing Medicine Ball Throw Test. In Squat and Vertical Jumping Tests, the "My Jump" application was used to determine the heights of the jumps [21]. In computing the anaerobic power by using jump height values, the formula developed by Johnson and Bahamonde

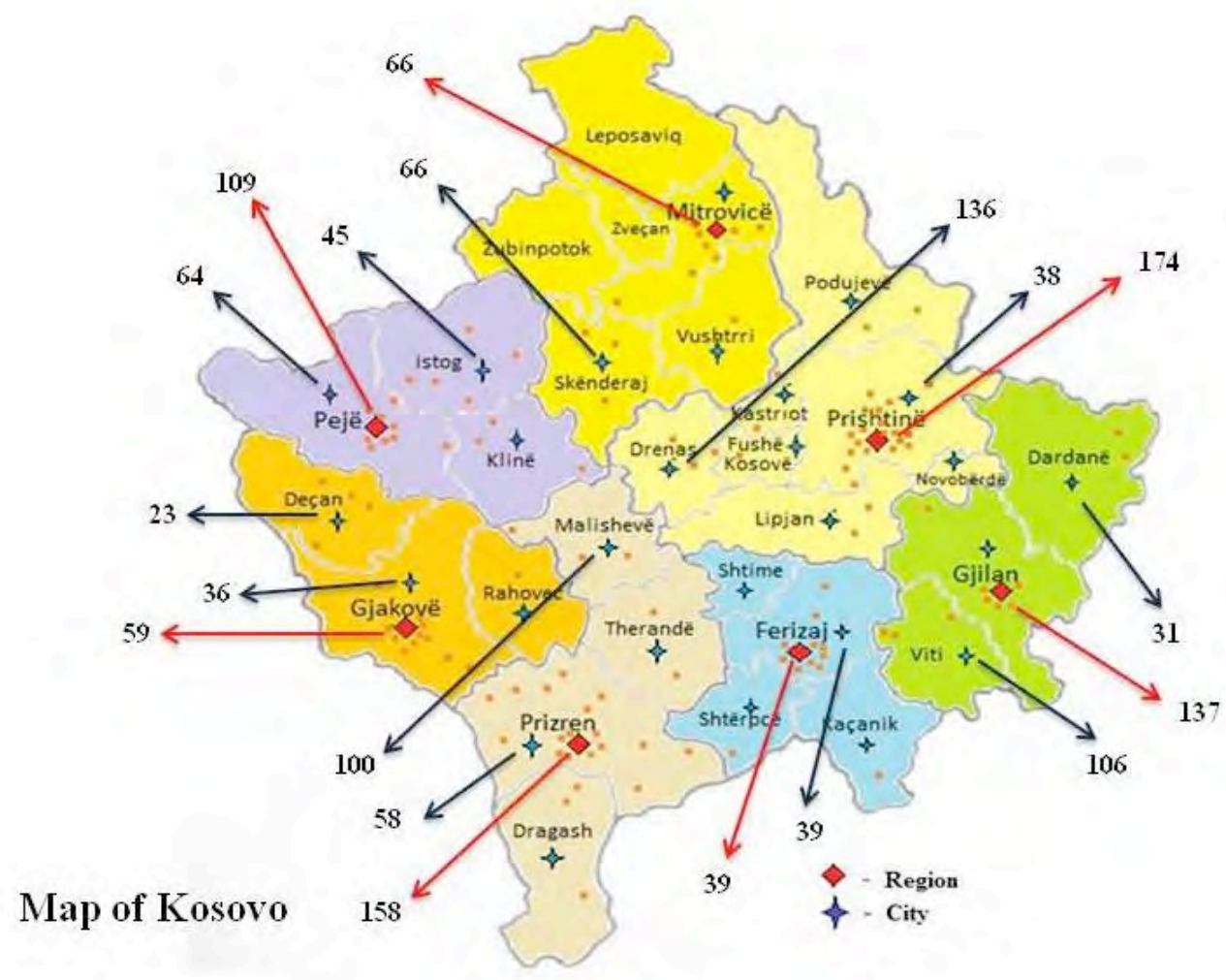

Figure 1. The regions and cities included in the sampling of the study 
was used [22]: Power-peak $(\mathrm{W})=(78.5 \cdot \mathrm{VJ}(\mathrm{cm})+60.3$ - mass $(\mathrm{kg})-15.3 \cdot$ height $(\mathrm{cm})-1308) /$ mass $(\mathrm{kg})$. In 30-meter speed test, an electronic chronometer (Seven Elektronik, SE-200) was used for measuring the time. To determine the maximal oxygen use capacity, the formula that computed the $\mathrm{VO}_{2} \max$ was used together with the Endurance Shuttle Run Test developed by Johnson and Bahamonde (1996) was employed [23]:

$\mathrm{VO}_{2} \max =3.46 *(\mathrm{~L}+\mathrm{SN} /(\mathrm{L} * 0.4325+7.0048))+12.2$

The Analysis of the Data

In computing the average, standard deviation, and percentage values of the physical and motor values of the study group, the SPSS 22 Program was employed. For the purpose of determining the percentages in norm values, the following percentage values, which are accepted as the Canadian Standard, were used. The scores below 20\% value were taken as "Very Low"; the scores between 21$40 \%$ range were taken as "Low"; the scores between 41 $60 \%$ range were taken as "Normal"; the scores between $61-80 \%$ range were taken as "High", and the scores above $81 \%$ were taken as "Very High" [24].

\section{Results}

In table 1, the norm values of the anthropometric characteristics, age and gender variables of the participants were determined. When the norm values were examined, $\mathrm{H}, \mathrm{W}$, and BMI values of 11-17 year-old girls and boys were increased paralle to the age increase. In addition, $\mathrm{BF} \%$ decreased depending on the age of the increase in both genders. When the mean values are examined, it is observed that there are gender-related differences.

In Table 2, the age and gender-specific norm values of the variables included in the participants' Eurofit test battery were determined. When the norm values were examined, the FBT, SAR, SUP, and PLT of 11-17 year-old girls and boys were increased parallel to the age increase. In addition, SHR values increased depending on the age of the increase in both genders. When the mean values of motor ability are examined, it is observed that there are gender-related differences.

In Table 3, the age and gender-specific norm values of the variables included in the participants' Eurofit test battery were determined. When the norm values were examined, the SBJ, HGR, BAH, and ESHR of 11-17 year-old girls and boys were increased parallel to the age increase. When the mean values of motor tests are examined, it is observed that there are gender-related differences.

Table 1. Heigh, Body Weight, Body Fat Percentage, Body Mass Index Normative Values

\begin{tabular}{|c|c|c|c|c|c|c|c|c|c|c|c|c|c|}
\hline & \multirow{2}{*}{ 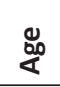 } & \multicolumn{2}{|l|}{$\overline{\mathrm{X}} \pm \mathrm{SD}$} & \multicolumn{2}{|c|}{ Very Low } & \multicolumn{2}{|l|}{ Low } & \multicolumn{2}{|c|}{ Normal } & \multicolumn{2}{|l|}{ High } & \multicolumn{2}{|c|}{ Very High } \\
\hline & & Girls & Boys & Girls & Boys & Girls & Boys & Girls & Boys & Girls & Boys & Girls & Boys \\
\hline \multirow{7}{*}{$\frac{\widehat{\underline{E}}}{\underline{I}}$} & 11 & $1,50 \pm 0,07$ & $1,48 \pm 0,05$ & $<1,39$ & $<1,42$ & 1,40 & 1,43 & 1,45 & 147 & 1,50 & 1,51 & $>1,55$ & $>1,55$ \\
\hline & 12 & $1,55 \pm 0,08$ & $1,54 \pm 0,07$ & $<1,39$ & $<1,42$ & 1,40 & 1,43 & 1,48 & 1,49 & 1,56 & 1,55 & $>1,64$ & $>1,61$ \\
\hline & 13 & $1,60 \pm 0,05$ & $1,60 \pm 0,07$ & $<1,51$ & $<1,48$ & 1,52 & 1,49 & 1,58 & 1,55 & 1,64 & 1,61 & $>1,70$ & $>1,67$ \\
\hline & 14 & $1,60 \pm 0,04$ & $1,69 \pm 0,07$ & $<1,52$ & $<1,61$ & 1,53 & 1,62 & 1,58 & 1,68 & 1,63 & 1,74 & $>1,68$ & $>1,80$ \\
\hline & 15 & $1,61 \pm 0,06$ & $1,72 \pm 0,07$ & $<1,59$ & $<1,63$ & 1,60 & 1,64 & 1,65 & 1,70 & 1,70 & 1,76 & $>1,75$ & $>1,82$ \\
\hline & 16 & $1,65 \pm 0,04$ & $1,74 \pm 0,07$ & $<1,59$ & $<1,60$ & 1,60 & 1,61 & 1,65 & 1,69 & 1,70 & 1,77 & $>1,75$ & $>1,85$ \\
\hline & 17 & $1,67 \pm 0,07$ & $1,76 \pm 0,07$ & $<1,59$ & $<1,68$ & 1,60 & 1,69 & 1,66 & 1,76 & 1,72 & 1,83 & $>1,78$ & $>1,90$ \\
\hline \multirow{7}{*}{$\begin{array}{l}\frac{000}{2} \\
3 \\
3\end{array}$} & 11 & $39,8 \pm 10,77$ & $37,3 \pm 6,28$ & $<29,5$ & $<32,7$ & 29,6 & 32,8 & 37,5 & 37,1 & 45,4 & 41,4 & $>53,3$ & $>45,7$ \\
\hline & 12 & $44,5 \pm 9,78$ & $42,9 \pm 9,09$ & $<36,3$ & $<33,5$ & 36,4 & 33,6 & 45,3 & 41,1 & 54,1 & 48,6 & $>63,0$ & $>56,1$ \\
\hline & 13 & $50,0 \pm 7,94$ & $47,9 \pm 8,46$ & $<38,2$ & $<39,6$ & 38,3 & 39,7 & 46,7 & 48,4 & 55,1 & 57,1 & $>63,2$ & $>65,8$ \\
\hline & 14 & $51,4 \pm 0,39$ & $54,6 \pm 0,63$ & $<35,1$ & $<32,9$ & 35,2 & 33,0 & 43,4 & 43,0 & 51,6 & 53,0 & $>59,8$ & $>63,0$ \\
\hline & 15 & $54,4 \pm 7,28$ & $58,7 \pm 9,39$ & $<39,6$ & $<37,8$ & 39,7 & 37,9 & 46,3 & 48,8 & 52,9 & 59,7 & $>59,5$ & $>70,6$ \\
\hline & 16 & $58,3 \pm 6,84$ & $64,2 \pm 10,89$ & $<54,1$ & $<55,9$ & 54,2 & 56,0 & 60,2 & 67,0 & 66,2 & 78,0 & $>72,2$ & $>89,0$ \\
\hline & 17 & $56,8 \pm 6,52$ & $66,1 \pm 10,62$ & $<52,0$ & $<53,9$ & 52,1 & 54,0 & 57,7 & 64,2 & 63,3 & 74,4 & $>68,9$ & $>84,6$ \\
\hline \multirow{7}{*}{$\frac{\widehat{ఏ}}{\frac{0}{0}}$} & 11 & $22,7 \pm 6,50$ & $18,8 \pm 2,92$ & $<17,1$ & $<16,1$ & 17,2 & 16,2 & 22,4 & 18,4 & 27,6 & 20,6 & $>32,8$ & $>22,8$ \\
\hline & 12 & $21,7 \pm 4,78$ & $17,0 \pm 4,61$ & $<15,9$ & $<14,1$ & 16,0 & 14,2 & 20,0 & 18,4 & 24,0 & 22,6 & $>28,0$ & $>26,8$ \\
\hline & 13 & $22,8 \pm 3,89$ & $16,7 \pm 5,51$ & $<19,1$ & $<14,5$ & 19,2 & 14,6 & 22,4 & 20,2 & 25,6 & 25,8 & $>28,8$ & $>31,4$ \\
\hline & 14 & $24,3 \pm 5,42$ & $14,7 \pm 3,28$ & $<20,9$ & $<11,9$ & 21,0 & 12,0 & 27,0 & 15,0 & 33,0 & 18,0 & $>39,0$ & $>21,0$ \\
\hline & 15 & $23,2 \pm 4,37$ & $14,0 \pm 2,90$ & $<22,7$ & $<12,1$ & 22,8 & 12,2 & 26,6 & 15,4 & 30,4 & 18,6 & $>34,2$ & $>21,8$ \\
\hline & 16 & $23,2 \pm 4,64$ & $15,0 \pm 5,07$ & $<16,3$ & $<12,7$ & 16,4 & 12,8 & 21,8 & 19,6 & 27,2 & 26,4 & $>32,6$ & $>33,2$ \\
\hline & 17 & $22,3 \pm 5,74$ & $13,4 \pm 4,02$ & $<16,7$ & $<09,5$ & 16,8 & 09,6 & 21,6 & 13,2 & 26,4 & 16,8 & $>31,2$ & $>20,4$ \\
\hline \multirow{7}{*}{ 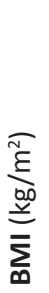 } & 11 & $17,1 \pm 3,89$ & $16,9 \pm 2,12$ & $<13,6$ & $<14,9$ & 13,7 & 15,0 & 16,3 & 16,2 & 18,9 & 17,4 & $>21,5$ & $>18,6$ \\
\hline & 12 & $18,3 \pm 3,32$ & $17,8 \pm 2,57$ & $<14,6$ & $<15,8$ & 14,7 & 15,9 & 17,6 & 18,3 & 20,5 & 20,7 & $>23,4$ & $>23,1$ \\
\hline & 13 & $18,9 \pm 2,94$ & $18,7 \pm 2,83$ & $<15,0$ & $<16,6$ & 15,1 & 16,7 & 17,7 & 19,7 & 20,3 & 22,7 & $>22,9$ & $>25,7$ \\
\hline & 14 & $19,7 \pm 2,73$ & $19,1 \pm 2,10$ & $<15,2$ & $<15,8$ & 15,3 & 15,9 & 17,8 & 18,4 & 20,3 & 20,9 & $>22,8$ & $>23,4$ \\
\hline & 15 & $20,1 \pm 2,75$ & $19,8 \pm 2,62$ & $<17,4$ & $<15,2$ & 17,5 & 15,3 & 20,4 & 18,6 & 23,3 & 21,9 & $>26,2$ & $>25,2$ \\
\hline & 16 & $21,2 \pm 2,79$ & $21,2 \pm 3,40$ & $<14,0$ & $<18,0$ & 14,1 & 18,1 & 17,6 & 22,3 & 21,1 & 26,5 & $>24,6$ & $>30,7$ \\
\hline & 17 & $21,2 \pm 2,25$ & $21,4 \pm 2,77$ & $<19,3$ & $<17,9$ & 19,4 & 18,0 & 21,0 & 20,7 & 22,6 & 23,4 & $>24,2$ & $>26,1$ \\
\hline
\end{tabular}

X̄: Mean, SD: Standard Deviation, H: Heigh, W: Body Weight, BF\%: Body Fat Percentage, BMI: Body Mass Index 
Table 2. Flamingo Balance Test, Sit and Reach, Sit Up, Plate Tapping, Shuttle Run 10x5 Meters Tests Normative Values

\begin{tabular}{|c|c|c|c|c|c|c|c|c|c|c|c|c|c|}
\hline & \multirow{2}{*}{$\underset{\text { 品 }}{ }$} & \multicolumn{2}{|l|}{$\bar{X} \pm S D$} & \multicolumn{2}{|c|}{ Very Low } & \multicolumn{2}{|l|}{ Low } & \multicolumn{2}{|c|}{ Normal } & \multicolumn{2}{|l|}{ High } & \multicolumn{2}{|c|}{ Very High } \\
\hline & & Girls & Boys & Girls & Boys & Girls & Boys & Girls & Boys & Girls & Boys & Girls & Boys \\
\hline \multirow{7}{*}{$\begin{array}{l}\overline{\tilde{o}} \\
\underline{0} \\
\underline{\Xi} \\
\text { - }\end{array}$} & 11 & $11,9 \pm 4,79$ & $11,8 \pm 8,35$ & $<8$ & $<7$ & 9 & 8 & 15 & 14 & 21 & 20 & $>27$ & $>26$ \\
\hline & 12 & $10,4 \pm 4,96$ & $9,5 \pm 5,58$ & $<5$ & $<5$ & 6 & 6 & 12 & 12 & 18 & 18 & $>24$ & $>24$ \\
\hline & 13 & $10,0 \pm 5,37$ & $9,4 \pm 4,28$ & $<5$ & $<3$ & 6 & 4 & 11 & 8 & 16 & 12 & $>21$ & $>16$ \\
\hline & 14 & $8,8 \pm 6,23$ & $9,4 \pm 4,63$ & $<4$ & $<3$ & 5 & 4 & 10 & 8 & 15 & 12 & $>20$ & $>16$ \\
\hline & 15 & $9,2 \pm 6,23$ & $9,7 \pm 6,09$ & $<4$ & $<5$ & 5 & 6 & 10 & 12 & 15 & 18 & $>20$ & $>24$ \\
\hline & 16 & $9,2 \pm 4,74$ & $8,9 \pm 6,51$ & $<4$ & $<7$ & 5 & 8 & 10 & 15 & 15 & 22 & $>20$ & $>29$ \\
\hline & 17 & $9,0 \pm 5,38$ & $7,5 \pm 6,27$ & $<4$ & $<4$ & 5 & 5 & 9 & 10 & 13 & 15 & $>17$ & $>20$ \\
\hline \multirow{7}{*}{$\frac{\widehat{\frac{\varepsilon}{U}}}{\frac{\alpha}{\tilde{\sigma}}}$} & 11 & $16,7 \pm 4,04$ & $14,9 \pm 5,15$ & $<9$ & $<7$ & 10 & 8 & 14 & 13 & 18 & 18 & $>22$ & $>23$ \\
\hline & 12 & $16,3 \pm 5,56$ & $15,5 \pm 5,21$ & $<10$ & $<7$ & 11 & 8 & 15 & 12 & 19 & 16 & $>23$ & $>20$ \\
\hline & 13 & $16,3 \pm 6,09$ & $14,2 \pm 5,83$ & $<11$ & $<7$ & 12 & 8 & 17 & 12 & 22 & 16 & $>27$ & $>20$ \\
\hline & 14 & $15,9 \pm 7,97$ & $14,8 \pm 6,86$ & $<7$ & $<10$ & 8 & 11 & 14 & 17 & 20 & 23 & $>26$ & $>29$ \\
\hline & 15 & $16,4 \pm 7,77$ & $15,0 \pm 8,09$ & $<8$ & $<7$ & 9 & 8 & 15 & 15 & 21 & 22 & $>27$ & $>29$ \\
\hline & 16 & $16,0 \pm 9,55$ & $14,5 \pm 8,34$ & $<2$ & $<1$ & 3 & 2 & 10 & 9 & 17 & 16 & $>24$ & $>23$ \\
\hline & 17 & $15,2 \pm 7,67$ & $13,8 \pm 8,31$ & $<7$ & $<2$ & 8 & 3 & 14 & 10 & 20 & 17 & $>26$ & $>24$ \\
\hline \multirow{7}{*}{ 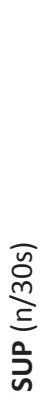 } & 11 & $15 \pm 3,43$ & $17 \pm 4,89$ & $<10$ & $<13$ & 11 & 14 & 13 & 18 & 15 & 22 & $>17$ & $>26$ \\
\hline & 12 & $17 \pm 4,88$ & $22 \pm 4,69$ & $<14$ & $<15$ & 15 & 16 & 19 & 20 & 23 & 24 & $>27$ & $>28$ \\
\hline & 13 & $18 \pm 3,76$ & $22 \pm 4,08$ & $<14$ & $<16$ & 15 & 17 & 17 & 20 & 19 & 23 & $>21$ & $>26$ \\
\hline & 14 & $19 \pm 3,09$ & $24 \pm 4,64$ & $<15$ & $<18$ & 16 & 19 & 18 & 22 & 20 & 25 & $>22$ & $>28$ \\
\hline & 15 & $21 \pm 4,62$ & $25 \pm 4,23$ & $<17$ & $<21$ & 18 & 22 & 21 & 26 & 24 & 30 & $>27$ & $>34$ \\
\hline & 16 & $21 \pm 4,81$ & $26 \pm 4,95$ & $<17$ & $<22$ & 18 & 23 & 21 & 26 & 24 & 29 & $>27$ & $>32$ \\
\hline & 17 & $28 \pm 3,66$ & $31 \pm 5,38$ & $<22$ & $<25$ & 23 & 26 & 25 & 30 & 27 & 34 & $>29$ & $>38$ \\
\hline \multirow{7}{*}{$\begin{array}{l}\bar{n} \\
\bar{a}\end{array}$} & 11 & $12,76 \pm 2,36$ & $14,45 \pm 2,00$ & $<11,18$ & $<09,92$ & 11,19 & 9,93 & 12,51 & 14,45 & 13,83 & 14,97 & $>15,15$ & $>17,49$ \\
\hline & 12 & $11,34 \pm 1,96$ & $11,57 \pm 2,57$ & $<07,41$ & $<08,17$ & 7,42 & 8,18 & 09,82 & 11,13 & 12,22 & 14,08 & $>14,62$ & $>17,03$ \\
\hline & 13 & $11,07 \pm 2,92$ & $11,25 \pm 2,31$ & $<07,42$ & $<07,93$ & 7,43 & 7,94 & 09,82 & 10,73 & 12,21 & 13,52 & $>14,60$ & $>16,31$ \\
\hline & 14 & $10,05 \pm 2,02$ & $10,27 \pm 2,48$ & $<07,37$ & $<07,57$ & 7,38 & 7,58 & 09,76 & 10,06 & 12,14 & 12,54 & $>14,52$ & $>15,02$ \\
\hline & 15 & $10,54 \pm 1,79$ & $10,02 \pm 1,06$ & $<08,46$ & $<06,61$ & 8,47 & 6,62 & 09,88 & 9,24 & 11,29 & 11,86 & $>12,70$ & $>14,48$ \\
\hline & 16 & $10,45 \pm 1,96$ & $10,01 \pm 1,62$ & $<09,35$ & $<08,94$ & 9,36 & 8,95 & 11,32 & 10,55 & 13,28 & 12,15 & $>15,24$ & $>13,75$ \\
\hline & 17 & $10,13 \pm 1,33$ & $9,87 \pm 1,44$ & $<09,36$ & $<08,23$ & 9,37 & 8,24 & 10,65 & 9,61 & 11,93 & 10,98 & $>13,21$ & $>12,35$ \\
\hline \multirow{7}{*}{ 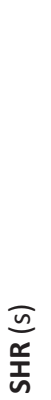 } & 11 & $22,42 \pm 1,19$ & $22,69 \pm 1,37$ & $<20,80$ & $<21,77$ & 20,81 & 21,78 & 21,65 & 22,64 & 22,49 & 23,50 & $>23,33$ & $>24,36$ \\
\hline & 12 & $22,75 \pm 1,43$ & $21,37 \pm 1,79$ & $<21,07$ & $<20,21$ & 21,08 & 20,22 & 22,40 & 21,92 & 23,72 & 23,62 & $>23,04$ & $>25,32$ \\
\hline & 13 & $22,78 \pm 1,47$ & $21,46 \pm 2,15$ & $<21,38$ & $<19,47$ & 21,39 & 19,48 & 22,66 & 22,21 & 23,93 & 24,94 & $>25,20$ & $>27,67$ \\
\hline & 14 & $22,01 \pm 1,74$ & $20,58 \pm 1,72$ & $<13,49$ & $<19,01$ & 13,50 & 19,02 & 17,00 & 20,72 & 20,50 & 22,42 & $>24,00$ & $>24,12$ \\
\hline & 15 & $23,89 \pm 2,82$ & $20,84 \pm 2,30$ & $<15,79$ & $<18,68$ & 15,80 & 18,69 & 18,43 & 20,88 & 21,06 & 23,07 & $>23,69$ & $>25,26$ \\
\hline & 16 & $24,59 \pm 2,49$ & $21,12 \pm 4,24$ & $<22,15$ & $<21,06$ & 22,16 & 21,07 & 23,94 & 28,14 & 25,72 & 35,21 & $>27,50$ & $>42,28$ \\
\hline & 17 & $24,06 \pm 3,85$ & $21,38 \pm 3,46$ & $<16,08$ & $<15,29$ & 16,09 & 15,30 & 19,96 & 18,48 & 23,83 & 21,66 & $>27,70$ & $>24,84$ \\
\hline
\end{tabular}

X: Mean, SD: Standart Deviation, FBT: Flamingo Balance Test, SAR: Sit and Reach, SUP: Sit Up, PLT: Plate Tapping, SHR: Shuttle Run 10x5 Meters 
Table 3. Standing Broad Jump, Hand Grip, Bent Arm Hang, 20 Meters Shuttle Run Tests Normative Values

\begin{tabular}{|c|c|c|c|c|c|c|c|c|c|c|c|c|c|}
\hline & \multirow[b]{2}{*}{$\stackrel{8}{⿺ 辶 一}$} & \multicolumn{2}{|l|}{$\overline{\mathbf{X}} \pm \mathrm{SD}$} & \multicolumn{2}{|c|}{ Very Low } & \multicolumn{2}{|l|}{ Low } & \multicolumn{2}{|c|}{ Normal } & \multicolumn{2}{|l|}{ High } & \multicolumn{2}{|c|}{ Very High } \\
\hline & & Girls & Boys & Girls & Boys & Girls & Boys & Girls & Boys & Girls & Boys & Girls & Boys \\
\hline \multirow{7}{*}{ 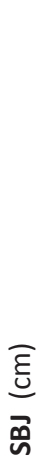 } & 11 & $1,29 \pm 0,24$ & $1,29 \pm 0,21$ & $<1,09$ & $<1,03$ & 1,10 & 1,04 & 1,30 & 1,25 & 1,50 & 1,46 & $>1,70$ & $>1,67$ \\
\hline & 12 & $1,38 \pm 0,20$ & $1,54 \pm 0,24$ & $<1,09$ & $<1,16$ & 1,10 & 1,17 & 1,29 & 1,39 & 1,48 & 1,61 & $>1,67$ & $>1,83$ \\
\hline & 13 & $1,37 \pm 0,23$ & $1,62 \pm 0,29$ & $<1,17$ & $<1,19$ & 1,18 & 1,20 & 1,36 & 1,45 & 1,54 & 1,70 & $>1,72$ & $>1,95$ \\
\hline & 14 & $1,54 \pm 0,22$ & $1,81 \pm 0,24$ & $<1,15$ & $<1,40$ & 1,16 & 1,41 & 1,32 & 1,65 & 1,48 & 1,89 & $>1,64$ & $>2,13$ \\
\hline & 15 & $1,57 \pm 0,18$ & $1,85 \pm 0,29$ & $<1,25$ & $<1,40$ & 1,26 & 1,41 & 1,41 & 1,64 & 1,56 & 1,87 & $>1,71$ & $>2,10$ \\
\hline & 16 & $1,53 \pm 0,26$ & $1,91 \pm 0,31$ & $<1,22$ & $<1,53$ & 1,23 & 1,54 & 1,42 & 1,88 & 1,61 & 22,2 & $>1,80$ & $>2,56$ \\
\hline & 17 & $1,60 \pm 0,29$ & $1,97 \pm 0,31$ & $<1,27$ & $<1,53$ & 1,28 & 1,54 & 1,46 & 1,88 & 1,64 & 2,22 & $>1,82$ & $>2,56$ \\
\hline \multirow{7}{*}{ 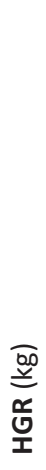 } & 11 & $17 \pm 5,24$ & $24 \pm 4,70$ & $<13$ & $<14$ & 14 & 15 & 18 & 19 & 22 & 23 & $>26$ & $>27$ \\
\hline & 12 & $20 \pm 5,13$ & $24 \pm 6,11$ & $<14$ & $<16$ & 15 & 17 & 19 & 22 & 23 & 27 & $>27$ & $>32$ \\
\hline & 13 & $25 \pm 5,15$ & $30 \pm 6,71$ & $<18$ & $<20$ & 19 & 21 & 23 & 27 & 27 & 33 & $>31$ & $>39$ \\
\hline & 14 & $30 \pm 6,71$ & $37 \pm 9,59$ & $<21$ & $<26$ & 22 & 27 & 29 & 35 & 36 & 43 & $>43$ & $>51$ \\
\hline & 15 & $29 \pm 6,17$ & $43 \pm 8,80$ & $<26$ & $<30$ & 27 & 31 & 34 & 39 & 41 & 47 & $>48$ & $>55$ \\
\hline & 16 & $30 \pm 5.85$ & $47 \pm 9,30$ & $<25$ & $<29$ & 26 & 30 & 31 & 39 & 36 & 48 & $>41$ & $>57$ \\
\hline & 17 & $31 \pm 5,84$ & $50 \pm 9,97$ & $<26$ & $<33$ & 27 & 34 & 34 & 45 & 41 & 56 & $>48$ & $>67$ \\
\hline \multirow{7}{*}{$\begin{array}{l}\frac{\pi}{T} \\
\frac{T}{\sigma}\end{array}$} & 11 & $3,84 \pm 1,02$ & $7,30 \pm 4,51$ & $<02,79$ & $<04,77$ & 02,80 & 04,48 & 03,60 & 08,08 & 04,40 & 11,38 & $>05,20$ & $>14,68$ \\
\hline & 12 & $4,89 \pm 3,26$ & $8,61 \pm 6,75$ & $<04,39$ & $<06,54$ & 04,40 & 05,55 & 07,80 & 12,10 & 11,20 & 17,65 & $>14,60$ & $>23,20$ \\
\hline & 13 & $4,62 \pm 2,52$ & $9,21 \pm 5,80$ & $<03,55$ & $<07,01$ & 03,56 & 07,02 & 06,12 & 11,04 & 08,68 & 15,06 & $>11,24$ & $>19,08$ \\
\hline & 14 & $5,35 \pm 4,66$ & $14,22 \pm 8,18$ & $<05,73$ & $<09,09$ & 05,74 & 09,10 & 10,48 & 16,10 & 15,22 & 23,10 & $>19,96$ & $>30,10$ \\
\hline & 15 & $5,55 \pm 2,16$ & $15,35 \pm 9,96$ & $<07,89$ & $<12,22$ & 07,90 & 12,23 & 09,22 & 12,41 & 10,54 & 32,59 & $>11,86$ & $>42,77$ \\
\hline & 16 & $7,91 \pm 3,06$ & $20,92 \pm 12,04$ & $<06,39$ & $<12,59$ & 06,40 & 12,60 & 08,80 & 24,08 & 11,20 & 35,56 & $>13,60$ & $>47,04$ \\
\hline & 17 & $7,86 \pm 6,26$ & $21,32 \pm 8,32$ & $<07,30$ & $<17,95$ & 07,31 & 17,96 & 12,62 & 25,92 & 17,93 & 33,88 & $>23,24$ & $>41,84$ \\
\hline \multirow{7}{*}{ 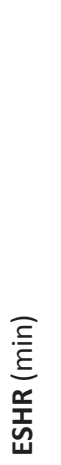 } & 11 & $3,2 \pm 0,73$ & $4,3 \pm 1,25$ & $<2 / 6$ & $<3 / 3$ & $2 / 7$ & $3 / 4$ & $3 / 2$ & $4 / 2$ & $3 / 7$ & $5 / 1$ & $>4 / 2$ & $>5 / 8$ \\
\hline & 12 & $3,2 \pm 1,41$ & $4,6 \pm 1,56$ & $<3 / 8$ & $<3 / 5$ & $4 / 1$ & $3 / 6$ & $5 / 6$ & $4 / 8$ & $7 / 3$ & $6 / 1$ & $>8 / 10$ & $>7 / 2$ \\
\hline & 13 & $3,6 \pm 0,95$ & $4,7 \pm 1,51$ & $<3 / 1$ & $<3 / 3$ & $3 / 2$ & $3 / 4$ & $3 / 7$ & $4 / 6$ & $4 / 4$ & $5 / 8$ & $>5 / 2$ & $>6 / 10$ \\
\hline & 14 & $3,4 \pm 0,71$ & $4,9 \pm 1,89$ & $<2 / 6$ & $<3 / 6$ & $2 / 7$ & $3 / 7$ & $3 / 4$ & $5 / 2$ & $4 / 2$ & $6 / 7$ & $>4 / 8$ & $>8 / 2$ \\
\hline & 15 & $3,2 \pm 0,99$ & $5,0 \pm 1,50$ & $<4 / 1$ & $<3 / 6$ & $4 / 2$ & $3 / 7$ & $4 / 9$ & $5 / 2$ & $5 / 6$ & $6 / 7$ & $>6 / 3$ & $>8 / 2$ \\
\hline & 16 & $3,5 \pm 0,99$ & $5,1 \pm 1,27$ & $<3 / 1$ & $<3 / 8$ & $3 / 2$ & $4 / 1$ & $4 / 2$ & $4 / 8$ & $5 / 1$ & $5 / 7$ & $>5 / 9$ & $>6 / 6$ \\
\hline & 17 & $3,8 \pm 1,42$ & $5,7 \pm 1,37$ & $<3 / 2$ & $<4 / 2$ & $3 / 3$ & $4 / 3$ & $4 / 5$ & $5 / 5$ & $5 / 7$ & $6 / 7$ & $>6 / 9$ & $>7 / 9$ \\
\hline
\end{tabular}

X: Mean, SD: Standart Deviation, SBJ: Standing Broad Jump, HGR: Hand Grip, BAH: Bent Arm Hang, ESHR: 20 Meters Shuttle Run 
Table 4. Standing Medicine Ball Throw, Arch Up Test, Push-up, Maximum Oxygen Intake Normative Values

\begin{tabular}{|c|c|c|c|c|c|c|c|c|c|c|c|c|c|}
\hline & \multirow[b]{2}{*}{$\stackrel{\text { 㟧 }}{\alpha}$} & \multicolumn{2}{|l|}{$\overline{\mathrm{X}} \pm \mathrm{SD}$} & \multicolumn{2}{|c|}{ Very Low } & \multicolumn{2}{|l|}{ Low } & \multicolumn{2}{|c|}{ Normal } & \multicolumn{2}{|l|}{ High } & \multicolumn{2}{|c|}{ Very High } \\
\hline & & Girls & Boys & Girls & Boys & Girls & Boys & Girls & Boys & Girls & Boys & Girls & Boys \\
\hline \multirow{7}{*}{$\begin{array}{l}\overline{\underline{\xi}} \\
\underline{\underline{I}} \\
\underline{\underline{\Sigma}}\end{array}$} & & $2,41 \pm 0,37$ & $2,97 \pm 0,39$ & $<2,16$ & $<2,51$ & 2,17 & 2,52 & 2,49 & 2,80 & 2,81 & 3,08 & $>3,13$ & $>3,36$ \\
\hline & & $2,74 \pm 0,57$ & $2,98 \pm 0,52$ & $<2,39$ & $<2,58$ & 2,40 & 2,59 & 2,80 & 3,13 & 3,20 & 3,67 & $>3,60$ & $>4,21$ \\
\hline & & $2,92 \pm 0,48$ & $3,54 \pm 0,72$ & $<2,55$ & $<2,68$ & 2,56 & 2,69 & 3,02 & 3,27 & 3,48 & 3,85 & $>3,94$ & $>4,43$ \\
\hline & & $3,01 \pm 0,50$ & $4,08 \pm 0,85$ & $<2,45$ & $<3,67$ & 2,46 & 3,68 & 2,92 & 4,51 & 3,38 & 5,34 & $>3,84$ & $>6,17$ \\
\hline & & $3,05 \pm 0,48$ & $4,47 \pm 0,89$ & $<3,17$ & $<3,69$ & 3,18 & 3,70 & 3,71 & 4,40 & 4,24 & 5,10 & $>4,77$ & $>5,80$ \\
\hline & & $3,17 \pm 0,41$ & $4,88 \pm 0,92$ & $<2,67$ & $<3,35$ & 2,68 & 3,36 & 3,06 & 4,22 & 3,44 & 5,08 & $>3,82$ & $>5,94$ \\
\hline & & $3,32 \pm 0,47$ & $5,01 \pm 0,87$ & $<3,23$ & $<3,85$ & 3,24 & 3,86 & 3,68 & 4,71 & 4,12 & 5,56 & $>4,56$ & $>6,41$ \\
\hline \multirow{7}{*}{$\begin{array}{l}\bar{n} \\
\frac{0}{2} \\
\frac{0}{2} \\
\frac{5}{2}\end{array}$} & 11 & $11 \pm 6,04$ & $14 \pm 7,05$ & $<06$ & $<11$ & 07 & 12 & 11 & 20 & 16 & 28 & $>20$ & $>36$ \\
\hline & 12 & $11 \pm 3,58$ & $15 \pm 9,52$ & $<07$ & $<12$ & 08 & 13 & 12 & 22 & 16 & 31 & $>20$ & $>40$ \\
\hline & 13 & $12 \pm 7,11$ & $17 \pm 9,13$ & $<09$ & $<12$ & 10 & 13 & 15 & 22 & 20 & 31 & $>25$ & $>40$ \\
\hline & 14 & $15 \pm 9,18$ & $18 \pm 7,76$ & $<12$ & $<12$ & 13 & 13 & 20 & 21 & 27 & 29 & $>34$ & $>37$ \\
\hline & 15 & $15 \pm 7,08$ & $18 \pm 8,47$ & $<12$ & $<14$ & 13 & 15 & 19 & 25 & 25 & 35 & $>31$ & $>45$ \\
\hline & 16 & $18 \pm 7,04$ & $19 \pm 8,90$ & $<16$ & $<12$ & 17 & 13 & 23 & 21 & 29 & 29 & $>35$ & $>37$ \\
\hline & 17 & $19 \pm 8,99$ & $23 \pm 11,71$ & $<16$ & $<18$ & 17 & 19 & 24 & 28 & 31 & 37 & $>38$ & $>46$ \\
\hline \multirow{7}{*}{ 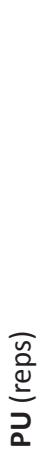 } & 11 & $6,5 \pm 3,63$ & $9,0 \pm 3,93$ & $<5$ & $<5$ & 6 & 6 & 9 & 9 & 12 & 12 & $>15$ & $>15$ \\
\hline & 12 & $5,7 \pm 3,64$ & $11,4 \pm 6,38$ & $<5$ & $<7$ & 6 & 8 & 9 & 12 & 12 & 16 & $>15$ & $>20$ \\
\hline & 13 & $5,7 \pm 3,07$ & $12,2 \pm 6,19$ & $<5$ & $<9$ & 6 & 10 & 9 & 15 & 12 & 20 & $>15$ & $>25$ \\
\hline & 14 & $6,2 \pm 2,66$ & $13,3 \pm 5,85$ & $<5$ & $<13$ & 6 & 14 & 9 & 19 & 12 & 24 & $>15$ & $>29$ \\
\hline & 15 & $7,1 \pm 1,96$ & $17,5 \pm 9,07$ & $<6$ & $<14$ & 7 & 15 & 8 & 22 & 9 & 29 & $>10$ & $>36$ \\
\hline & 16 & $7,3 \pm 2,36$ & $20,5 \pm 10,59$ & $<6$ & $<16$ & 7 & 17 & 9 & 25 & 11 & 33 & $>13$ & $>41$ \\
\hline & 17 & $9,2 \pm 2,94$ & $23,2 \pm 8,34$ & $<7$ & $<17$ & 8 & 18 & 10 & 26 & 12 & 34 & $>14$ & $>42$ \\
\hline \multirow{7}{*}{ 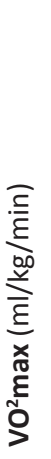 } & 11 & $23,6 \pm 2,49$ & $27,3 \pm 4,31$ & $<21,7$ & $<24,5$ & 21,8 & 24,6 & 25,6 & 27,5 & 25,4 & 30,4 & $>27,2$ & $>33,3$ \\
\hline & 12 & $23,2 \pm 3,43$ & $28,4 \pm 5,26$ & $<21,1$ & $<24,9$ & 21,3 & 25,0 & 24,7 & 29,2 & 28,1 & 33,4 & $>31,5$ & $>37,6$ \\
\hline & 13 & $24,9 \pm 3,15$ & $28,7 \pm 5,14$ & $<24,5$ & $<24,1$ & 24,6 & 24,2 & 28,8 & 28,4 & 33,0 & 32,6 & $>37,2$ & $>36,8$ \\
\hline & 14 & $24,3 \pm 2,40$ & $29,5 \pm 6,39$ & $<22,2$ & $<25,1$ & 22,3 & 25,2 & $24,6-$ & 30,4 & 26,9 & 35,6 & $>29,2$ & $>40,8$ \\
\hline & 15 & $23,6 \pm 3,36$ & $29,6 \pm 5,05$ & $<14,5$ & $<25,1$ & 14,6 & 25,2 & 17,0 & 30,4 & 19,4 & 35,6 & $>21,8$ & $>40,8$ \\
\hline & 16 & $24,6 \pm 3,39$ & $30,1 \pm 4,31$ & $<23,5$ & $<25,4$ & 23,6 & 25,5 & 26,8 & 28,5 & 30,0 & 31,5 & $>33,2$ & $>34,5$ \\
\hline & 17 & $25,7 \pm 4,80$ & $32,1 \pm 4,66$ & $<24,2$ & $<27,0$ & 24,3 & 27,1 & 29,1 & 31,2 & 33,9 & 35,3 & $>38,7$ & $>39,5$ \\
\hline
\end{tabular}

X: Mean, SD: Standart Deviation, MBTH: Standing Medicine Ball Throw, AUT: Arch Up Test, PU: Push-up, VO2max: Maximum Oxygen Intake 
Table 5. Squat Jump, Countermovement Jump, Maximal Anaerobic Power, 30 - Meters Speed Test Normative Values

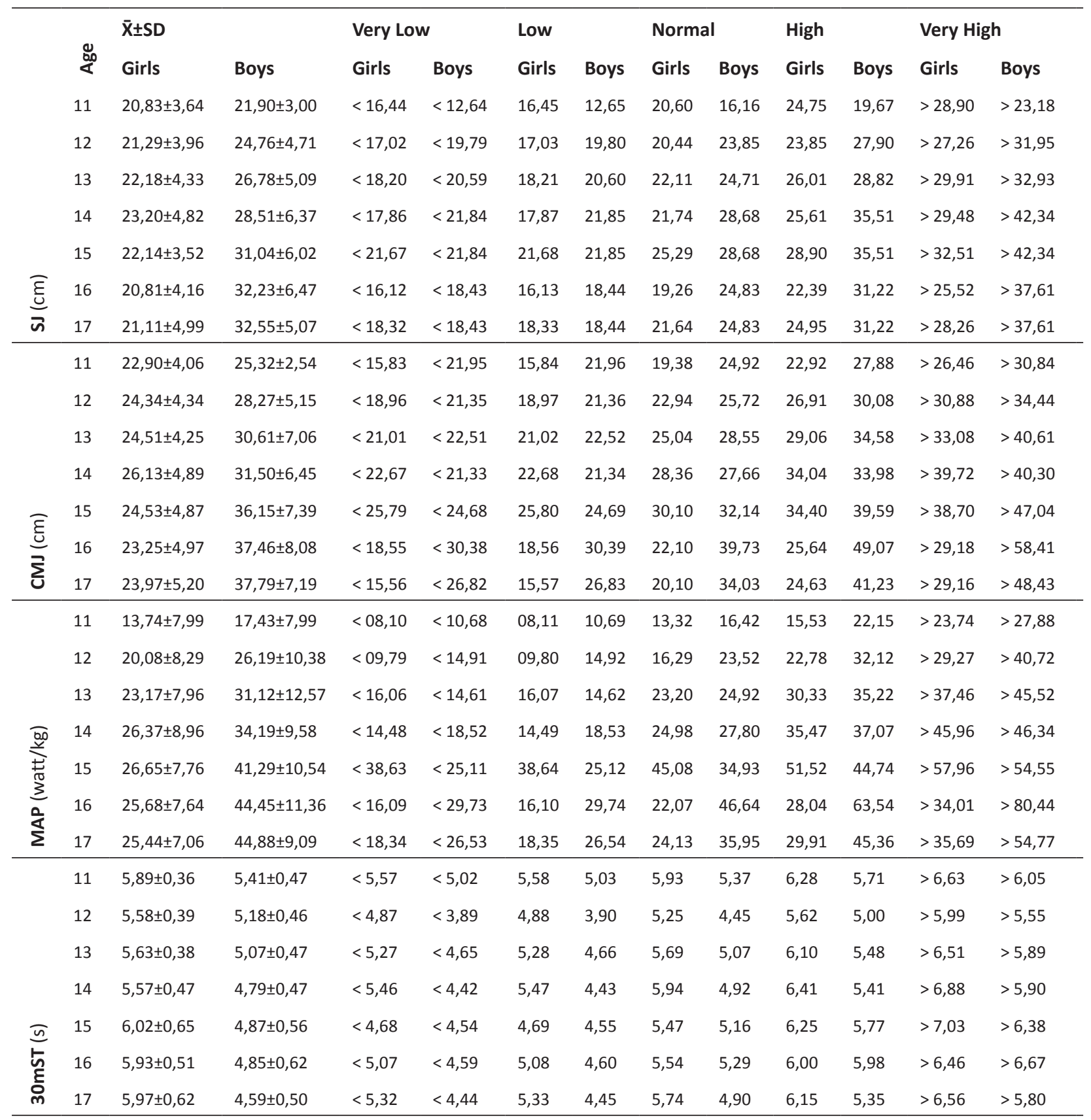

$\bar{X}$ : Mean, SD: Standart Deviation, SJ: Squat Jump, CMJ: Countermovement Jump, MAP: Maximal Anaerobic Power, 30mST: 30 - Meters Speed Test

In Table 4, the age and gender-specific norm values of the variables included in the participants' motoric tests were determined. When the norm values were examined, the MBTH, AUT, PU, and $\mathrm{VO}_{2 \max }$ of 11-17 year-old girls and boys were increased linearly with the age increase. When the mean values of motor tests are examined, it is observed that there are gender-related differences.

In Table 5, the age and gender-specific norm values of the variables included in the participants' motoric tests were determined. When the norm values were examined, the SJ, CMJ, MAP, and 30mST of 11-17 year-old girls and boys were increased parallel to the age increase. When the mean values of motor tests are examined, it is observed that there are gender-related differences.

\section{Discussion}

In the anthropometric, physical and motoric values obtained in the study, it was observed that there were differences depending on the gender variable, which is consistent with the literature data $[6,25,26]$. It was observed that the female students in Kosovo were taller and heavier than their male peers until the age of 13, which is the case in their peers in other countries as well. Similarly, according to Spurway and MacLearn (2007), the growth spurt occurs generally at the age of 12 in females, and around the age of 14 in males [27]. Again 
consistent with the literature data, it was observed in the present study that the female students had higher Body Fat Percentage values at each age group when compared with the male students included in the study. When the differences observed in developmental periods of children depending on age were analyzed, it was determined that the height and body weight values increased in a parallel manner to the increasing age in both genders, which is consistent with the literature data. It is known that the heights of males exceeded the heights of females 2-3 years after the puberty, and the period in which the growth speed was at the highest level was observed between 9-15 years of age in females, and between 11-17 years of age in males [28]. When the height values were compared with those defined by the World Health Organization (WHO), it was observed that the male and female students in Kosovo had higher values than the world average values in each age group [26]. It was also determined in the present study that the BMI values show similarities with those reported by WHO.

When the FLB Test Values, which was used in assessing the balance ability, were analyzed, it was observed that the Balance Ability improved with the increasing age, which was reported by Miguel et al. [29]. The balance scores obtained in the study were compared with the norm values reported by Tomkinson et al. after 123655 measurements in 19 countries. It was observed that the normal values of the male and female students in Kosovo were worse balance ability scores according to the values in the 50 percentile reported in the study conducted by Tomkinson except for 13 and 14 years of age [6]. Again, the balance scores were compared with the balance scores obtained by Jürimäe \& Volbekiene (1998) in Estonia in students from similar age groups. It was observed that the 12-17-year-old males and 11-12-year-old and 14-year-old females living in Kosovo had higher performances when compared with those living in Estonia; and the 11-yearold males and 13 and 15-17-year-old females had lower performances [11].

When the values obtained in the Arm Movement Speed test, which was used for the purpose of assessing the upper extremity speeds, were analyzed, it was observed that the arm movement speed improved depending of furthering age. It was determined that the improvement speed was lower in females between 14-15 years of age, and also lower in males between 15-16 years of age. According to Piotr et al. (2007), it was observed that the kinesthetic perception, muscle internal coordination and inter-muscular coordination were disrupted in puberty period [30]. In addition to these, it is also well-known that speed and coordination properties are affected by anthropometric properties of individuals [31]. The arm movement speeds obtained in the study were compared with the norm values reported by Tomkinson et al. after 148093 measurements in 19 countries in Europe. It was observed that the normal values in arm movement speeds of the male and female students in Kosovo were lower than those reported by Tomkinson in 50 percentile [6]. Similarly, again our scores were compared with the scores reported by Jürimäe and Volbekiene in Estonia. It was observed that the males and females living in Kosovo had better values than the children in Estonia [11]. The scores of the Sit and Reach Flexibility Test, which gives us data on the flexibility property of the whole body, were evaluated. It was observed that the female students in Kosovo were more flexible than the males at all age groups in Kosovo, which is consistent with the literature data $[5,28]$. When the changes in the SAR values were analyzed according to age, it was observed that the flexibility ability decreased in both genders parallel to the increasing age. Similar results on the changes depending on age were also reported by Kjaer et al. [32]. On the other hand, it was also observed that the female and male children living in Kosovo were less flexible when compared with the children living in Estonia [11] and Australia [5]. When compared with the norm values reported by Tomkinson et al. after their 464807 measurements in 27 countries in Europe, it was observed that the male and female students in Kosovo had lower flexibility properties [6].

When the scores obtained in power tests were analyzed it was observed that there were changes that were consistent with the literature data. It was also observed that the male students living in Kosovo were more successful than the female students in all power tests. In addition, increases were observed in power values depending on the increase in age. It is known that together with the chronologic age, anaerobic power increases at a constant rate in lower and upper extremities until young adulthood as of 10 years of age [33-35].

When the test values obtained in the Standing Long Jump, which gives us data on elastic power, and which is used frequently, were compared with the norm values reported after 464900 measurements in 29 countries in Europe, it was observed that the values of the male and females in Kosovo were lower [6]. While the value reported by Tomkinson et al. (2017) for 50 percentile was $156.4 \mathrm{~cm}$ for females at the age of 17 , it was $205.8 \mathrm{~cm}$ for 17-year-old males; these values were determined as $146 \mathrm{~cm}$ for female students and $188 \mathrm{~cm}$ for male students living in Kosovo. Similarly, it was observed that the male and female students living in Estonia were more successful than their peers living in Kosovo [11]. In addition, according to the study conducted by Alexander et al. (2016) on children living in Colombia and India, the 11-17-year-old male and female children living in these countries had lower values than those living in Kosovo [36].

When the scores of the SJ and CMJ tests, which are used in assessing the explosive and elastic power, were analyzed, it was observed that the explosive and elastic power improved with the increasing age, which was also reported by Çonkaz (2017). These scores increased between the age of 11 and 14 in females; it was observed that they decreased after this age limit $[34,35]$. It was also observed that the male students living in Kosovo were more successful than the female students in both test values [25, 37]. The scores of the SJ and CMJ tests, which give information on the explosive and elastic power of 
the legs were compared with the other countries. It was observed when compared with the scores reported by Ortega et al. (2011) after making 3528 measurements in 9 countries in Europe that the scores of the male and female students in Kosovo are higher [25].

When the Hand Grip Force test, which is one of the upper extremity force tests, values were compared, it was observed that the male students living in Kosovo had lower values than their peers living in Europe in each age group. On the other hand, it was also determined that the female students in Kosovo had higher values in Hand Grip Force when compared with their peers in Europe [6].

While the normal value for the 17-year-old female students in Kosovo was $34 \mathrm{~kg}$, the Hand Grip Force value for 50 percentile was determined as $28 \mathrm{~kg}$ for 24 countries in Europe in 203295 measurements by Tomkinson et al. (2017). Again, in Bent Arm Hang Test, which is one of the upper extremity force tests, the values determined for female and male students in Kosovo were higher than their counterparts in Europe. The normal value for 17-year-old female students in Kosovo was $12.62 \mathrm{~s}$ and for male students $25.92 \mathrm{~s}$. It was reported by Tomkinson et al. (2017) after 189673 measurements in 23 countries in Europe that the Bent Arm Hang Test value for 50 percentile in females was $6.16 \mathrm{~s}$ and $24.46 \mathrm{~s}$ for males [6].

When the results of the sit-up and arch-up test results, which are used in assessing the body flexor and extensor muscle power, were analyzed, it was observed that the values of the male students were higher than the values of the female students, which is the case in all power tests. Again as expected, increases were detected in force values parallel to the increase in the age. It was also observed that the male and female students in Kosovo are unsuccessful in sit-up test, and had similar values when compared with their peers in Europe for further ages. The sit-up values obtained in the study were compared with those reported by Tomkinson et al. (2017) in 481032 measurements in 23 countries in Europe. 25 repetitions for females, and 30 repetitions for males were determined for Kosovo. In European norm values, the same value was 25 for male students and 20 for female students [6].

It was also observed that males had higher values than females in the ESHR and $\mathrm{VO}_{2}$ max values, which provide data on general endurance properties [38]. It was observed that endurance increased with furthering age; however, there appeared a decrease in females between the ages of 13 and 15, and it decreased with puberty. According to a study conducted by Coleman and Hendry (1999), the development speed of endurance changes in puberty period [39]. The ESHR Test values were compared with the norm values reported by Tomkinson et al. (2017) after 445092 measurements in 24 countries in Europe. This value was determined as 4.5 for females and 5.5 for males in Kosovo. In European norm values, the value was 4.11 for females who are 17 years old, and 7.66 for males who are 17 years old [6]. Again, it was observed that the children living in Kosovo had lower ESHR Test values when compared with their peers in Estonia [11]. Similar to the ESHR test results, it was also determined that the
VO2max values computed for male and female students in Kosovo were lower than the children between 11-17 years of age living in Colombia and India [36], and the children between 11-12 years of age in Turkey [40].

\section{Conclusions}

It is considered that the norm values of the male and female children between the ages of 11-17 living in Kosovo determined in the present study by using the results obtained may be used in follow-up of the physical and motoric properties and developments of children at the same age group. These norm values may be used in the decision-making processes about the physical status and weak and strong sides of children. The students whose strong sides are defined may be directed to proper sports branches. Very low values may be used to determine proper fitness targets, to follow-up long-term changes, to encourage positive behaviors about health, and to determine whether there are serious health problems. In addition to these, the defined norm values will also make contributions in comparing the children in developmental periods living in Kosovo with their peers living in other countries. It may be recommended to re-plan the Physical Education and Sports classes applied in Kosovo for the purpose of improving the properties like the endurance properties that were found to be lower in children living in Kosovo when compared with other countries. In this context, the norm values determined in the 21 physical and motoric tests will be shared with the Ministry of Education, Science and Technology, sports institutions and clubs.

As a conclusion, while the children living in Kosovo showed higher performances in the tests that were affected by anthropometric properties like height and body fat percentage and by genetic properties like the speed of the movements of the arms when compared with the children from other countries from the same age group; they had lower performances in the tests that were affected by life style and physical activity levels like endurance, long jump, and flexibility.

\section{Acknowledgment}

This work was supported by the EBDTEZ-2016-70-02-003 project file and by the Sakarya University Scientific Research Projects Coordination Unit.

Physical education and Sports teachers and Test Team who contributed to the data collection:

Agim Berisha, Almir Berisha, Ardit Berisha, Leotrim Elshani, Mejdi Hoti, Valon Kastrati, Agron Thaqi, Kastriot Kafexholli, Liridon Telaku, Veton Telaku, Kadri Krasniqi, Fisnik Lushtaku, Binaze Hyseni-Tërshani, Albert Nikqi, Arton Haxhiaj, Ferdane Knushi, Alban Bojku, Egzon Nezaj, Ardita Berisha, Adrian Telaku, Mirkend Linjani, Sadik Ferati, Lumi Ademaj, Hasan Bytyqi.

\section{Conflict of interests}

The authors declare that there is no conflict of interests. 


\section{References}

1. Dictionary.com [Internet]. 2016 [cited 07.12.2016]. Available from: http://www.dictionary.com/browse/norm? $\mathrm{s}=\mathrm{t}$

2. English Oxford living dictionary [Internet]. American and British Academy. 2016 [cited 07.12.2016]. Available from: https://en.oxforddictionaries.com/definition/norm

3. Lopes VP, Stodden DF, Bianchi MM, Maia JAR, Rodrigues LP. Correlation between BMI and motor coordination in children. Journal Science and Medicine in Sport. 2012;15:3843.

4. Zorba E, Saygın Ö. Fiziksel Aktivite ve Fiziksel Fygunluk [Physical Activity and Physical Fitness]. İstanbul: 2008. (In Turkish).

5. Catley M, Tomkinson G. Normative health-related fi tness values for children: analysis of 85347 test results on 9-17-year-old Australians since 1985. Sports Medicine. 2011;1:1-12.

6. Tomkinson G, Carver K, Atkinson F, Daniell N, Lewis L, Fitzgerald J. European normative values for physical fitness in children and adolescents aged 9-17 years: results from 2779165 Eurofit performances representing 30 countries. British Journal of Sports Medicine. 2017;1:1-13.

7. Balyi I, Way R, Higgs C, Norris S, Cardinal C. Canadian Sport for Life In: Institute CS, editor. Canadian Sport for Life - Long-Term Athlete Development Resource Paper 20. Pacific: Canadian Sport Institute; 2014. p. 81.

8. Popović R, Aleksić A, Stojanović D, Stefanović M, Božić S, Popović M. Evaluation of the Physical Fitness Level in Physical Education Female Students Using "EurofitTest”. International Journal of Sports Science and Physical Education. 2017;2(1):1-15.

9. Hergüner G, Önal A, Berisha M, Yaman MS. The Comparison of Physical Education and Sports Lessons Applied in Education Systems of Turkey and Kosovo. Universal Journal of Educational Research, 2016;4(9): 1985-1993.

10.Ordinary Plans and Programs 1-13. Kosovo: Ministry of Education, Science and Technology; 2017. (In Albanian).

11.Jürimäe T, Volbekiene V. Eurofit Test Results in Estonian and Lithuanian 11 to 17-Year-Old Children: A Comparative Study. European Journal of Physical Education. 1998;3(2):178184.

12.Asmussen E, Klissouras V, Tuxworth B, Lofi A, Leger L. Sport testing physical Eurofit In: Karaesk F, editor. Testing physical fitness Eurofit Experimental Battery. Strasbourg Council of Europe; 1983. p. 1-83.

13. Council of Europe. Of the committee of ministers to member states on the Eurofit tests of physical fitness. Ministers CoECo: Europe Council of Europe Committee of Ministers; 1987.

14.Kemper H, Mechelen W. Physical Fitness Testing of Children: A European Perspective. Pediatric Exercise Science: Human Kinetics Pubiishers, 1996;8:2010-2014.

15. Nhantumbo L, Saranga S, Prista A, Basso L, Maia J. Allometric study of functional fitness of children and adolescents in a rural area of Mozambique. Brazilian Journal of Kinanthropometry. 2012;14(5):508-516.

16. Wilczewski A, Sklad M, Krawczyk B, Saczuk J, Majle B. Physical Development and Fitness of Children from Urban and Rural Areas as Determined by EuroFit Test Battery. Biology of Sports. 1996;13(2):113-126.

17.Agjensia e Statistikave të Kosovës [Kosovo Agency of Statistics]. Kosova: Kosovo Agency of Statistics; [Internet]. 2014. [cited 07.12.2017]. Available from: http://ask.rks-gov. net/en/kosovo-agency-of-statistics. (In Albanian)

18.Mekolli E, Bruqi S, Lahi H, Rexhepi N, Fazliu M, Radoni S.
Education Statistics in Kosovo. Ministry of Education SaT: Republic of Kosovo; 2016.

19. Council of Europe. Committee for the development of sport. Eurofit: handbook for the EUROFIT tests of physical fitness: Council of Europe: Committee for the developement of sport; 1988.

20.Özer MK. Fiziksel uygunluk [Physical fitness]. Ankara: Nobel academic publishing education consultancy; 2016. (In Turkish).

21.Fernández C, Glaister M, Lockey R. The validity and reliability of an iPhone app for measuring vertical jump performance. Journal of Sports Sciences. 2015;33(15):15741579 .

22.Johnson DL, Bahamonde R. Power Output Estimate in University Athletes. Journal of strength and Conditioning Research. 1996;10(3):161-166.

23.Johnson DL, Bahamonde R. Power Output Estimate in University Athletes. Journal of Strength \& Conditioning Research. 1996;10(3):161-166.

24.Ed B. Exercise Physiology Laboratory Manual. USA WCB/ McGraw-Hill; 1998.

25.Ortega F, Artero E, Ruiz J, Espana-Romero V, JimenezPavon D, Vicente-Rodriguez G. Physical fitness levels among European adolescents: the HELENA study. $\mathrm{Br} J$ Sports Medicine. 2011;45:20-29.

26. World Health Organization [Internet]. World Health Organization: World Health Organization; 2017 [cited 2017 17.07.2017]. Available from: http://www.who.int/ childgrowth/en

27.Spurway N, MacLaren D. Pediatric Exercise Phisiology. Elsevier; 2007.

28.Yalnız Fİ, Oral O. Antrenman bilgisi ve sporcu să̆lı̆̆ [Training information and athlete health]. Ankara: Nobel academic publishing education consultancy; 2016. (In Turkish).

29.Miguel-Etayo PD, Gracia-Marco L, Ortega FB, Intemann T, Foraita R, Lissner L. Physical fitness reference standards in European children: the IDEFICS study. International Journal of Obesity. 2014;38:S57-S66.

30.Piotr U, Miguel C. Review of modern teaching methods for tennis. Revista internacional de ciencias del deporte / International Journal of Sport Science. 2007;7(3):1-10.

31.Amstrong N, Welsman JR, Williams CA, Kibry BJ. Longitudinal changes in young people's short-term power output. Medicine \& Science in Sports \& Exercise. 2000;32(6):1140-5.

32.Kjær I, Torstveit MK, Kolle E, Hansen BM, Anderssen SA. Normative values for musculoskeletal- and neuromotor fitness in apparently healthy Norwegian adults and the association with obesity: a cross-sectional study. BMC Sports Science, Medicine and Rehabilitation. 2016;8(37):1-9.

33.Doré E, Bedu M, França NM, Praagh EV. Anaerobic cycling performance characteristics in prebubescent adolescent and young adult females. European Journal of Applied Physiology. 2001;84:476-481.

34.Çonkaz H. Psikomotor gelişim - doğum öncesinden ölüme kadar [Psychomotor Development - From Birth to Death). Ankara: Gazi Kitabevi Tic. Ltd. Şti.; 2017. (In Turkish).

35.Bompa T, Haff G. Dönemleme; Antrenman kuramı ve yöntemi [Periodization; Theory and Methodology of Training]. Ankara; 2015. (In Turkish).

36.Alexander J, Sepúlveda R, Vélez RR, Bautista JEC, Izquierdo M, Hermoso AG. Physical fitness and anthropometric normative values among Colombian-Indian schoolchildren. MBP Public Health. 2016;16(962):2-15. 
37.Örjan E, Kristjan O, Björn E. Physical performance and body mass index in Swedish children and adolescents. Scandinavian Journal of Nutrition. 2005;49(4):172-179.

38.Tomkinson G, Lang J, Tremblay M, Dale M, LeBlanc A, Belanger K. International normative $20 \mathrm{~m}$ shuttle run values from 1142026 children and youth representing 50 countries. British journal of sports Medicine, 2016;1:1-14.
39.Coleman J, Hendry LB. The Nature of Adolosence. London and New York: Routelge; 1999.

40.Erikoğlu G, Özkamcı H, Golmoghanı N, Suveren C, Tot T, Şahin N. 7-12 yaş çocuklarda cinsiyet ve yaş gruplarına göre eurofit test bataryası ile performans parametrelerinin değerlendirilmesi [Evaluation of Eurofit test battery and performance parameters according to gender and age groups in 7-12 year old children]. Journal of Gazi Physical Education and Sport Sciences 2009;14(4):49-64. (In Turkish).

\section{Information about the authors:}

Milaim Berisha (Corresponding author); http://orcid.org/0000-0002-0353-7247; milaimberisha3@gmail.com; Institute of Educational Sciences, Faculty of Sports Science, Sakarya University; Sakarya University Esentepe Campus 54187 Serdivan / SAKARYA, Turkey.

Murat Çilli; http://orcid.org/0000-0002-9027-363X; mcilli@gmail.com; Department of Coaching Education, Faculty of Sports Science, Sakarya University; Sakarya University Esentepe Campus 54187 Serdivan / SAKARYA, Turkey.

Cite this article as: Milaim Berisha, Murat Çilli. Normative values for physical fitness in children aged 11-17 in Kosovo. Pedagogics, psychology, medical-biological problems of physical training and sports, 2018;22(1):17-27. doi:10.15561/18189172.2018.0103

The electronic version of this article is the complete one and can be found online at: http://www.sportpedagogy.org.ua/index.php/PPS/issue/archive

This is an Open Access article distributed under the terms of the Creative Commons Attribution License, which permits unrestricted use, distribution, and reproduction in any medium, provided the original work is properly cited (http://creativecommons.org/licenses/by/4.0/deed.en).

Received: 11.12.2017

Accepted: 30.12.2017; Published: 26.02.2018 